\title{
Temperature dependent elastic repulsion of colloidal nanoparticles with a polymer adsorption layer
}

\author{
Anatoly P. Gavrilyuk 1,2 . Valeriy S. Gerasimov 1,3 . \\ Alexander E. Ershov ${ }^{1,3}$. Sergey V. Karpov ${ }^{4,3,5}$
}

Received: date / Accepted: date

\begin{abstract}
The model of pairwise elastic repulsion of contacting colloidal nanoparticles with a rigid core and deformable shell is discussed. A simple analytical equation is applied for the energy of elastic repulsion of nanoparticles with arbitrary sizes and the elasticity moduli of self-healing polymer adsorption layers. The model is based on the representation of the absorption layer as a continuous medium that is elastically deformed upon the contact of nanoparticles. The major characteristic of this medium is the elasticity modulus. The magnitude of the elasticity modulus is determined from the condition of balance of the van der Waals attractive forces of nanoparticles and the elastic repulsion of their adsorption layers in the contact area, taking into account the temperature variations. We employed the kinetic approach to describe the dependence of the elasticity modulus on both the temperature and the rate of its change.
\end{abstract}

Keywords Nanoparticle · adsorption layer · elastic deformation · coagulation kinetics · elasticity modulus

\footnotetext{
Anatoly P. Gavrilyuk

E-mail: glyukonat@icm.krasn.ru

1 Institute of Computational Modeling SB RAS, 660036, Krasnoyarsk, Russia

2 Institute of Mathematics and Computer Science, Siberian Federal University, Krasnoyarsk, 660041, Russia

${ }^{3}$ Laboratory of Nanotechnology, Spectroscopy and Quantum Chemistry, Siberian Federal University, Krasnoyarsk, 660041, Russia

4 Kirensky Institute of Physics, Federal Research Center KSC SB RAS, 660036, Krasnoyarsk, Russia

5 Siberian State University of Science and Technology, Krasnoyarsk 660014, Russia
}

\section{Introduction}

The synthesis of nanocolloids with particular properties and high coagulation stability is one of the important problems in production of pharmacological medications in the form of hydrosols with a long storage life [16], nanocolloids-based nonlinear-optical limiters [7-9], printer ink [10] and etc.

A water-soluble polymer is frequently introduced into the solution of initial reagents before chemical synthesis of nanoparticles begins [11-15] in order to prevent coagulation and subsequent sedimentation of the disperse phase. In this case, each synthesized nanoparticle adsorbs polymer molecules and becomes surrounded by an adsorption layer (adlayer). The thickness of this polymer shell usually varies from $0.5 \mathrm{~nm}$ to $3 \mathrm{~nm}$ [16] while polymer tails can extend up to $\approx 23 \mathrm{~nm}[17,18]$. Adlayers prevent direct contact of nanoparticle cores in the case of their collision. Thus, the gap between the cores is around $1-5 \mathrm{~nm}$. The gap size affects both the structural and optical characteristics of an aggregate $[13,14,19,20]$, especially in the case of plasmonic nanocolloids. For example, pulsed laser irradiation of a plasmonic nanoparticle aggregate leads to local changes in its structural and spectral properties [21-27] and to its selective photomodification [24-27]. Local structural changes are related to the heating of resonant particles and their adlayer by radiation. The mechanical properties of the adlayer change under heating, which unbalances the elastic and the van der Waals forces. These effects are accompanied by relative displacement of the heated particles, which can produce selective variations in the structure of aggregates and their absorption spectra $[22]$.

There is a number of physical models that describe elastic interaction between nanoparticles with polymeric 
adlayers [20, 22, 28-32] . However, application of some of these models $[14,30,33-35]$ requires a proper choice of tuning parameters for specific types of polymer molecules. The lack of these data gives rise to inaccuracies in the calculation of elastic forces. In addition, the adequacy of these models becomes inappropriate in the case of polymers that form a sufficiently rigid spatial network (in particular, casein, gelatin and polyvinylpyrrolidone), which are often used in polymer-stabilized colloidal systems. The impact of high temperature and changes of mechanical properties of heated polymeric adlayers is a complex problem within the framework of the abovementioned models. Therefore, the development of a sufficiently simple and adequate temperature dependent physical model for the elastic interaction of colloidal particles in a liquid interparticle medium (especially using spatial network polymers) is of particular interest and importance.

Examples of the models of elastic interactions were presented in Refs $[28,29]$. These models were used for studying the conditions for crystallization of nanocolloids as well as interaction of metallic nanocolloids with a pulsed laser radiation. Our paper is devoted to a more detailed formulation and further elaboration of the model of elastic interaction taking into consideration the role of a solid core under a polymer shell with a different ratio of the undeformable core size and the adlayer thickness. The presented model allows both calculation of elastic forces between nanoparticles and their change in the process of heating or cooling, which is essential in studying the interaction of high-intensity pulsed laser radiation with nanoparticle aggregates.

\section{Model}

Let us consider the basic principles of the model with reference to a casein adlayer. One of the dominant factors that determines motion within a pair of bound particles under laser irradiation is the van der Waals attraction force. This attraction is mainly compensated by elastic forces due to the presence of an adlayer on the surface of nanoparticles. The following important assumption is used to describe these forces arising upon contact of adlayers: the adlayer is elastic and its mechanical properties are characterized by elasticity modulus (EM). The structural features of a polymer are manifested through the EM magnitude.

We can use the contact problem of two touching spheres $[34,36]$ to describe elastic interaction between two nanoparticles coated with a polymer adlayer, known as the Hertz contact problem. Figure 1 shows contact of two nanoparticles of the same radius $r_{0}$ and the same adlayer thickness $h_{0}$. The adsorbed layers are elastically deformed when they come into contact as the result of the van der Waals attraction so their thickness at the contact spot decreases to $h<h_{0}$, which gives rise to the elastic forces $F_{e l}$.

The potential energy of such an elastic interaction can be described by the following equation obtained by Hertz for macroscopic bodies [36]:

$$
\begin{gathered}
U_{e l}=\frac{8}{15}\left(h_{01}+h_{02}-h_{12}\right)^{5 / 2} \times \\
{\left[\frac{\left(r_{01}+h_{01}\right)\left(r_{02}+h_{02}\right)}{\left(r_{01}+h_{01}\right)+\left(r_{02}+h_{02}\right)}\right]^{1 / 2} \times} \\
\frac{E_{e l 1} \cdot E_{e l 2}}{E_{e l 1}\left(1-\sigma_{e l 2}^{2}\right)+E_{e l 2}\left(1-\sigma_{e l 1}^{2}\right)},
\end{gathered}
$$

where $E_{e l 1}$ and $E_{e l 2}$ are the adlayer elasticity moduli, $\sigma_{e l 1}, \sigma_{e l 2}$ are the adlayer Poisson ratios (characteristic value $\approx 0.15), h_{12}=2 h$. Considering particles of the same radius $\left(r_{01}=r_{02}=r_{0}\right)$ and the same adlayer thickness $\left(h_{01}=h_{02}=h_{0}\right)$, it can be assumed that the elasticity modulus and the Poisson ratio will also be the same $\left(E_{e l}=E_{e l 1}=E_{e l 2}, \sigma_{e l}=\sigma_{e l 1}=\sigma_{e l 2}\right)$ :

$U_{e l}=\frac{4}{15}\left(2 h_{0}-h_{12}\right)^{5 / 2}\left[\frac{r_{0}+h_{0}}{2}\right]^{1 / 2} \frac{E_{e l}}{1-\sigma_{e l}^{2}}$,

where $\sigma_{e l}$ is the adlayer effective Poisson ratio, $E_{e l}$ is the adlayer effective elasticity modulus. Taking into account the smallness of $\sigma_{e l}^{2}$, we can neglect this parameter in the future.

In contrast to the classical Hertz problem related to deformation of two balls in the contact area, in our case inside the spheres there is a rigid metallic core which at least at the initial stage does not affect the deformation process.

Let us now consider the process of coagulation of two particles (up to the moment of balance of forces) under the action of the van der Waals force $F_{v}$, the elastic force $F_{e l}=-d U_{e l} / d h_{12}$ and the viscous friction force $F_{f}$ (the description of all forces acting on particles is given in [29]). Fig. 2 shows qualitative dependence of the total interaction energy of the particles $U=U_{v}+U_{e l}$ on the distance between their surfaces (gap). Here $U_{v}$ is the van der Waals interaction energy. For description we use the approximation of the Hamaker and de Boer theory (see, for example [37]). According to this theory, the interaction energy of two spherical particles with radii $r_{01}$ and $r_{02}$ is described by the following equation: 
Fig. 1

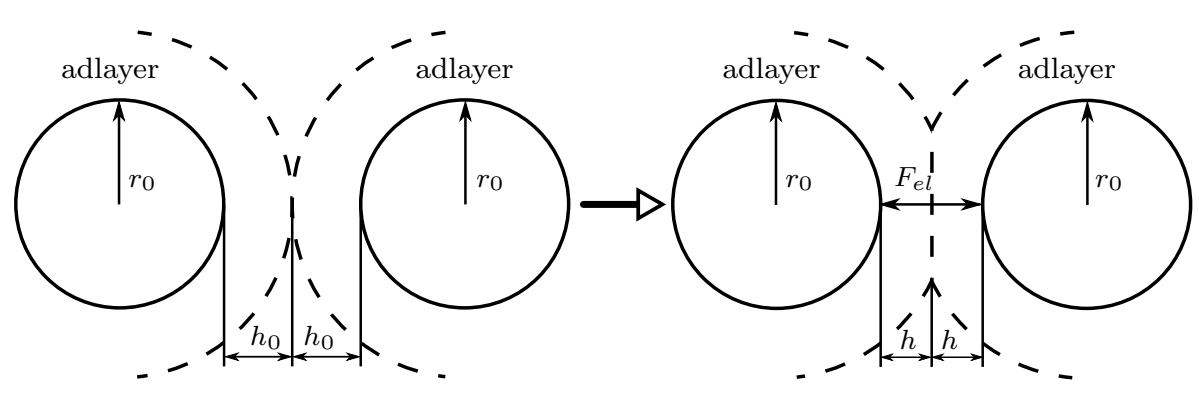

Contact deformation of adlayers of two identical particles.

$$
\begin{aligned}
U_{v} & =-\frac{A_{H}}{6}\left[\frac{2 r_{01} \cdot r_{02}}{h_{12}^{2}+2 h_{12}\left(r_{01}+r_{02}\right)}+\right. \\
& +\frac{2 r_{01} \cdot r_{02}}{h_{12}^{2}+2 h_{12}\left(r_{01}+r_{02}\right)+4 r_{01} \cdot r_{02}}+ \\
& \left.+\ln \frac{h_{12}^{2}+2 h_{12}\left(r_{01}+r_{02}\right)}{h_{12}^{2}+2 h_{12}\left(r_{01}+r_{02}\right)+4 r_{01} \cdot r_{02}}\right] .
\end{aligned}
$$

For equal-sized particles we have the equation:

$$
\begin{aligned}
U_{v}= & -\frac{A_{H}}{6}\left[\frac{2 r_{0}^{2}}{h_{12}^{2}+4 h_{12} r_{0}}+\frac{2 r_{0}^{2}}{h_{12}^{2}+4 r_{0} h_{12}+4 r_{0}^{2}}+\right. \\
& \left.\ln \left(\frac{h_{12}^{2}+4 h_{12} r_{0}}{h_{12}^{2}+4 r_{0} h_{12}+4 r_{0}^{2}}\right)\right],
\end{aligned}
$$

where $A_{H} \approx 50 k_{B} T_{0}$ is the effective Hamaker constant $[13,38,39]$, where $T_{0}=300 K$ and $k_{B}$ is the Boltzmann constant. Thus, the van der Waals attractive force that acts on each of the particles is equal to $F_{v}=-d U_{v} / d h_{12}$. The particles approach each other until the moment when their adlayers come into contact. This movement is prevented only by the viscous friction force $F_{f}$, and after the contact (the point $h_{12}^{0}$ in Fig. 2) - also by the elastic force $F_{e l}$.

As a result of approach of the particles, a stable state of a dimer with the distance $h_{12}^{s}$ between the particle surfaces is reached when the secondary potential well in Fig 2 satisfies the following conditions:

$$
U_{\max }-U_{\min } \geq k_{B} T,\left|U_{\min }\right| \geq k_{B} T \text {. }
$$

Figure 3 shows discrete values of the elasticity modulus vs the adlayer thickness $\left(h_{0}\right)$ for particles of different radii found numerically under condition

$U_{\max }-U_{\min } \approx k_{B} T$.

Note that for small particles $\left(r_{0}=5 \mathrm{~nm}\right)$, condition (6) is not satisfied when the adlayer thickness is $h_{0} \geq 2 \mathrm{~nm}$. That is, a system of two small particles with a thick adlayer is unstable and uncouples due to thermal fluctuations.

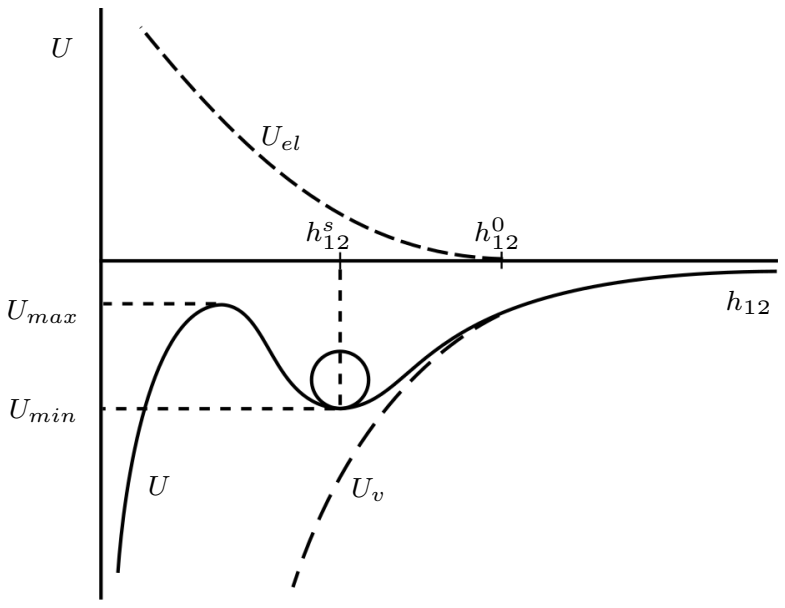

Fig. 2

The scheme of capture of a particle into a potential well.

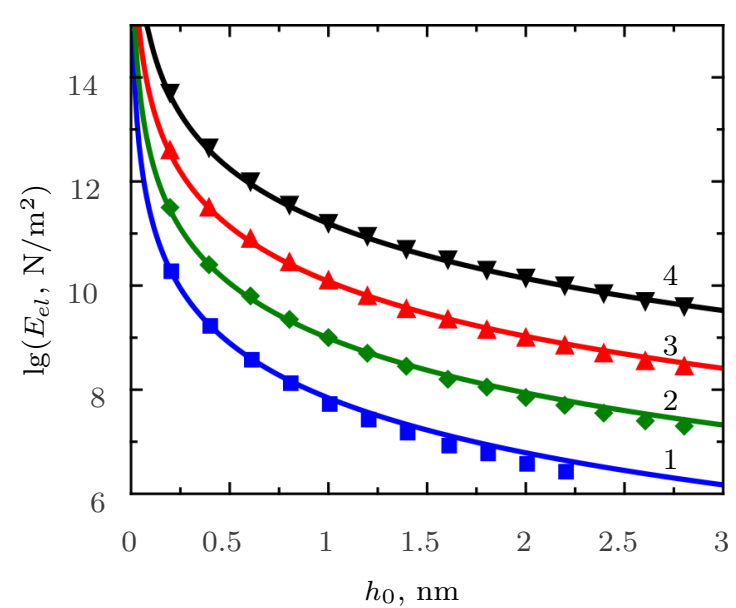

Fig. 3

Dependence of the effective elasticity modulus of a polymer adlayer on its thickness (points are numerical values, solid lines are approximations for particles with different radii: $1-r_{0}=5 \mathrm{~nm}, 2-r_{0}=10 \mathrm{~nm}, 3-r_{0}=15 \mathrm{~nm}, 4-$ $r_{0}=25 \mathrm{~nm}$. For ease of perception, curves 2, 3 and 4 are successively shifted upwards by one vertical scale step. 
To solve the problem, we use, in particular, data for polymer-stabilized silver hydrosols with particles having an adsorption layer of casein. These colloids were used in the experiments on photostimulated aggregation of metal hydrosols [39]. It turned out that the values of the elasticity modulus of the particle adlayer material corresponding to the condition (6) exceeded the values $E_{e l} \sim 10^{4} \mathrm{~N} / \mathrm{m}^{2}$ for the free casein gel by $4-5$ orders of magnitude (see [40]). The use of data from [40] in calculations does not help to explain the existence of nanoparticle aggregates with the interparticle gaps $h_{12} \simeq 1.0-5 \mathrm{~nm}$ when the elastic force and the van der Waals force would be balanced.

A significant difference in the elasticity modulus of the polymer adlayer of nanoparticles in comparison with the free gel can be substantiated by the following considerations. First, it is known [41] that in polymer films of nanometer thickness the value of the elasticity modulus $\left(E_{e l}\right)$ is significantly increased, which is associated with a decrease in the concentration of breaks of initial bonds (nodes) in the polymer spatial network.

Second, the increased adlayer elasticity modulus can be explained by attraction of polymer molecules to the surface of the particle due to image forces as well as electrostatic, van der Waals, ion-dipole, and other types of forces. That is, under such conditions, polymers in the adlayer are in a compressed state, which increases its density in comparison with the free gel.

In addition, due to interaction with the particle surface the gel structure changes, since the binding energy of the surface with polymer molecules is greater than the binding energy between the molecules themselves. The smaller the thickness of the polymer adlayer, the greater the contribution of short-range interactions of molecules with the surface of the metal core and hence the more compressed the adlayer and the higher the value of elasticity modulus.

To obtain an equation approximating $E_{e l}\left(h_{0}\right)$, let us consider equilibrium of nanoparticles under the condition:

$\frac{r_{0}}{h_{0}} \gg 1$.

In this case, the equations for the van der Waals energy and elastic interactions can be simplified:

$U_{v} \approx-\frac{A_{H}}{6}\left[\frac{r_{0}}{4 h}+\frac{1}{2}+\ln \left(\frac{2 h}{r_{0}}\right)\right]$,

$U_{e l}=\frac{4}{15}\left(2 h_{0}-2 h\right)^{5 / 2}\left(\frac{r_{0}}{2}\right)^{1 / 2} E_{e l}$.

Since in equilibrium the van der Waals force balances the elastic force $\left(\mathbf{F}_{v}+\mathbf{F}_{e l}=0\right)$, then using equation (8), we obtain:

$\frac{A_{H}}{48} \frac{r_{0}}{h^{2}}=\frac{8}{3} E_{e l} r_{0}^{1 / 2}\left(h_{0}-h\right)^{3 / 2}$.
It follows from this equation that to balance the forces, the elasticity modulus $E_{e l}$ must satisfy the condition:

$E_{e l} \geq \frac{A_{H}}{132} \cdot \frac{r_{0}^{1 / 2}}{h^{2}\left(h_{0}-h\right)^{3 / 2}}$.

Differentiating equation (10) with respect to $h$, we find that the value of the elasticity modulus is limited by the minimum magnitude, which is attained at $h=$ $4 h_{0} / 7$ and equals

$E_{\text {el min }}=0.083 A_{H}\left(\frac{r_{0}^{1 / 2}}{h_{0}^{7 / 2}}\right)$.

Next, assuming that the sought magnitude $E_{e l} \sim$ $E_{e l \text { min }}$ and choosing a coefficient that best satisfies the numerical values $E_{e l}$ for $A_{H} \approx 50 k_{B} T_{0}[13,38]$ (for gold and silver hydrosols) and $T_{0}=300 \mathrm{~K}$, we have:

$E_{e l} \approx 3.1 \cdot 10^{7}\left(\frac{r_{0}}{h_{0}^{7}}\right)^{1 / 2}$,

here $r_{0}$ and $h_{0}$ are given in $\mathrm{nm}$, and $E_{e l}$ in $\mathrm{N} / \mathrm{m}^{2}$. As it can be seen from Fig. 3, there is a good agreement between the numerical values and the theoretical approximation (solid lines).

Note that knowing the value of EM depending on the particle size and the initial thickness of the adlayer and using more general equations for the energies of elastic and van der Waals interactions (1) and (3), it is easy to find the interaction energy of two differently sized particles. An example of such calculation is shown in Figure 4.

2.1 Features of the elastic interaction of colloidal particles with a rigid core

Figure 5 shows a comparison of the dependences of the total van der Waals and elastic interaction energy on the interparticle gap. The elastic energy was calculated using both the Hertz model [36] and the finite elements method (FEM). FEM is widely used for numerical solving various elasticity problems [42]. In this figure, the magnitude of $E_{e l}$ was chosen so that the positions of the potential energy minima calculated by the above two models coincide. The figure shows that the depth of the potential well differs insignificantly. This allows us to conclude that the Hertz model can be used subject to a proper choice of the elasticity modulus. However in the case of large deformation of adlayers we must take into consideration amendments in the classical model related to the effect of rigid core. A detailed analysis of elastic interactions of spherical particles with 


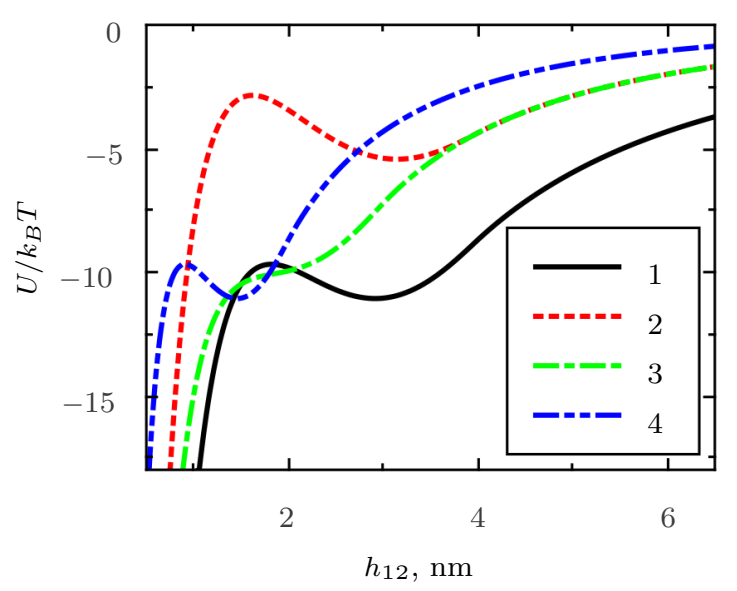

Fig. 4

The dependence of the potential energy of interaction of two nanoparticles of different radii $\left(r_{01}, r_{02}\right)$ and the initial thickness of the adlayers $\left(h_{01}, h_{02}\right)$ on the interparticle distance $h_{12}: 1-r_{01}=20 \mathrm{~nm}, h_{01}=2.0 \mathrm{~nm}, r_{02}=20 \mathrm{~nm}$, $h_{02}=2.0 \mathrm{~nm} ; 2-r_{01}=20 \mathrm{~nm}, h_{01}=2.0 \mathrm{~nm}, r_{02}=10 \mathrm{~nm}$, $h_{02}=2.0 \mathrm{~nm} ; 3-r_{01}=20 \mathrm{~nm}, h_{01}=2.0 \mathrm{~nm}, r_{02}=10 \mathrm{~nm}$, $h_{02}=1.0 \mathrm{~nm} ; 4-r_{01}=10 \mathrm{~nm}, h_{01}=1.0 \mathrm{~nm}, r_{02}=10 \mathrm{~nm}$, $h_{02}=1.0 \mathrm{~nm}$.

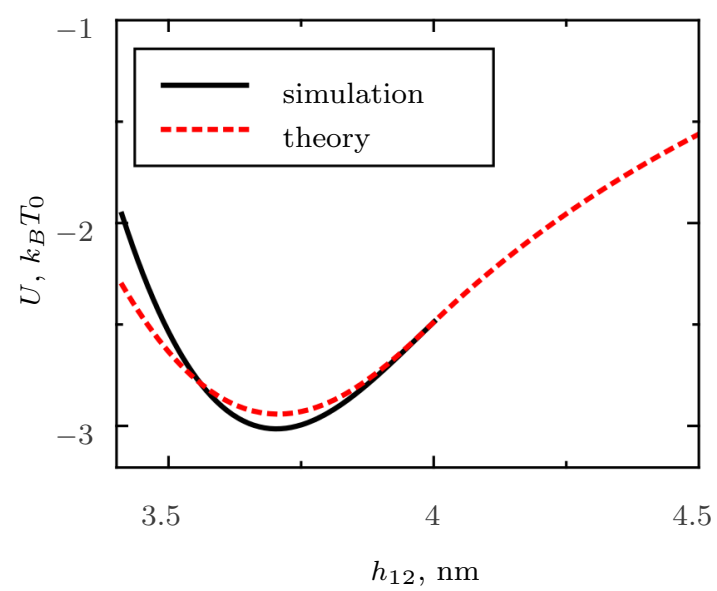

Fig. 5

Dependence of the total van der Waals and elastic interaction energy on interparticle gap. The energy of elastic interaction was calculated using equation (4) (dashed line) and by the finite elements method FEM (solid line). The van der Waals interaction energy in both cases was calculated with equation (4) using following parameters: $E_{e l}=10^{7} \mathrm{~N} / \mathrm{m}^{2}$ (dashed line), $E_{e l}=2.07 \cdot 10^{7} \mathrm{~N} / \mathrm{m}^{2}$ (solid line), $r_{0}=10 \mathrm{~nm}$, and $h_{0}=2 \mathrm{~nm}$.

deformable shells made by the finite elements method in a wide range of deformations will be published in a separate communication.

\section{Kinetics of the temperature dependent elasticity modulus of an adsorption layer}

The conditions for variation of the elasticity modulus with growing temperature arise, in particular, when colloids interact with optical radiation. So when highintensity pulsed laser radiation is applied to a colloid, considerable heating of the particles as well as of the polymer adlayer occurs. Under such conditions, intermolecular bonds break in the polymer network of the gel, which leads to a decrease in its elasticity modulus. This, in turn, disbalances the forces for the dimer particles because of the decreased elastic force. The latter leads to disappearance of the secondary potential well and intermediate barrier. In this case the particles begin approaching each other until their metal surfaces come into almost complete contact, that is, get into the main potential minimum.

Thus, temperature dependence of the elasticity modulus is an important factor that determines the ability of our model to reproduce the experimental results. For example, the value of EM for a free gel decreases from $1.3 \cdot 10^{4} \mathrm{~N} / \mathrm{m}$ to $3 \cdot 10^{3} \mathrm{~N} / \mathrm{m}$ with an increase in temperature by $30 \mathrm{~K}[40,43]$ (see Table 1$)$.

Table 1

Temperature dependence of the elasticity modulus of casein gel.

\begin{tabular}{lllll}
\hline$T, \mathrm{~K}$ & 280 & 293 & 310 & 323 \\
$E_{e l} \cdot 10^{-3}, \mathrm{~N} / \mathrm{m}^{2}$ & 13.6 & 7.9 & 3.3 & 2.1 \\
\hline
\end{tabular}

However, in our case, such a strong dependence is not observed in real experiments. Thus, the Ag hydrosol with isolated particles did not change its stability when heated up to $370 \mathrm{~K}$, which is confirmed by data on the absorption spectra [39]. This means that the mechanical (elastic) characteristics of the polymer particle adlayer in aggregates change only slightly in such a range with growing temperature, which can be explained by their strong initial compression and higher binding energy of polymer molecules with the particle surface.

Another important factor that determines the magnitude of the elasticity modulus of a polymer adlayer is the heating rate. In order to take this factor into account, we will consider variation of the elasticity modulus under heating not as an instantaneous process, but rather as the process occurring within a finite time. A similar kinetic approach was used earlier to describe the strength and distraction of solids (including polymers) [44-46].

To describe this process, it is convenient to relate the elastic properties of a polymer to its structure, which 
includes the sites of binding in polymer chains (nodes) where intermolecular interactions of their intersecting parts occur. We can assume that the elasticity modulus is proportional to the concentration of nodes $n$ in the spatial polymer network: $E_{e l} \sim n$ [47]. Then the decrease in the value of EM during heating is determined by ruptures of the network nodes and by destruction of its structure. The average node destruction time within which it can occur is finite and temperature dependent. This time decreases with growing temperature. The elasticity modulus will not change if during the time $\tau_{p}$ of the laser pulse duration and heating of the metal core no ruptures of nodes occur in adlayer. To determine the characteristic time of destruction of a node, we will use the Frenkel theory [48], according to which the mean waiting time $\tau_{f}$ for local fluctuations of energy (on one atom or a small group of atoms bound at the network site) is found as:

$\tau_{f} \approx \tau_{0} \cdot \exp \left(E_{f} / k_{B} T\right)$

Here $\tau_{0} \approx 10^{-12} \div 10^{-13} \mathrm{~s}$ is the characteristic time of vibrations of atoms in the polymer chain. Destruction of bonds with the potential barrier $U_{s}$ is possible, if $E_{f} \geq U_{s}$. The average waiting time for such an act is

$\tau_{d} \approx \tau_{0} \cdot \exp \left(U_{s} / k_{B} T\right)$,

which is the average characteristic time of rupture of the nodes or the time of relaxation (under heating) of the polymer to a new stable state (with a smaller number of bonds between the polymer molecules) corresponding to the changed temperature.

In our opinion, it is more convenient to describe the change in EM with temperature through the frequency of fluctuations $\nu_{f}$ :

$\nu_{f}(T)=\tau_{d}^{-1}=\tau_{0}^{-1} \exp \left(-U_{s} / k_{B} T\right)$.

Then the change $E_{e l}$ can be expressed by the equation

$\frac{d E_{e l}}{d t}=-\nu_{f}(T) E_{e l}$.

Equation (16) takes into account only the decay of the spatial structure of the polymer adlayer. However, along with disintegration, there must be an inverse process - the restoration of links (nodes). Only when both processes are taken into consideration we can describe relaxation of $E_{e l}$ to its equilibrium value and determine its characteristic time, which is especially important for describing relaxation of adlayers at the cooling stage of nanoparticles. Therefore, to describe the behavior of the elasticity modulus, a new term is added to equation (16), responsible for the reconstruction of an adlayer:

$\frac{d E_{e l}}{d t}=-\nu_{f}(T) E_{e l}+K_{r} E_{0}^{2 / 3} T\left(E_{0}-E_{e l}\right)$,

where $E_{0}$ is the elasticity modulus of the nondestructured heated gel, and $K_{r}$ is a constant coefficient. The values of $U_{s} E_{0}$ and $K_{r}$ will be determined below. A qualitative justification for the form of the second relaxation term in (17) is as follows.

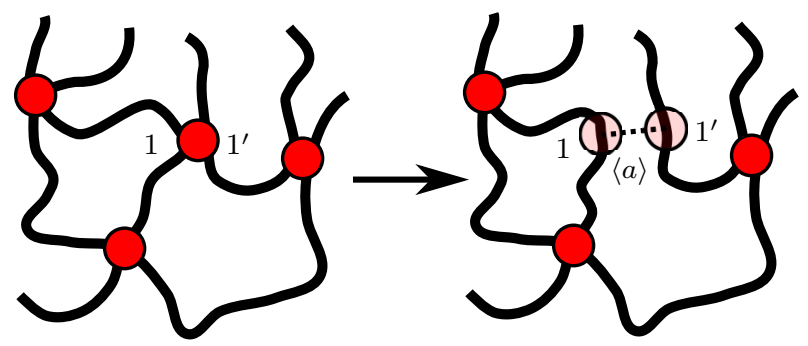

Fig. 6

Local section of a polymer network with a broken bond 1 $1^{\prime}$ (node).

Let $n_{0}$ be the maximum possible concentration of nodes in a nondestructured polymer network of adlayer, which in a destructured network decreases to the value of $n$ as a result of heating. Accordingly, concentration of the destroyed nodes will be $n_{0}-n$, then the probability of their association will be proportional to $\left(n_{0}-n\right)$. In addition, consider the motion of parts of the broken bonds (light circles in Figure 6) as one-dimensional motion of Brownian particles along the line of rupture $1-1^{\prime}$. Then using the Einstein formula, which determines mean displacement of a Brownian particle during the time $t$, we can estimate the characteristic time $\tau_{b}$ of displacement of parts of the node by an average interstitial distance $\langle a\rangle \approx n_{0}^{-1 / 3}$ :

$\tau_{b} \sim \frac{\langle a\rangle^{2}}{T}=\frac{1}{n_{0}^{2 / 3} T}$.

Proceeding from the assumption that the elasticity modulus is proportional to the number of nodes in the polymer network we can define the rate of its restoration in the following form:

$\frac{d E_{e l}}{d t} \sim \frac{1}{\tau_{b}}\left(n_{0}-n\right) \sim E_{0}^{2 / 3} T\left(E_{0}-E_{e l}\right)$,

which is reflected in equation (17). The constant coefficients $U_{s}, K_{r}$ and $E_{0}$ (see equation (17)) can be found from the experimental values of the elasticity modulus obtained for different fixed temperatures (see Table 1). 


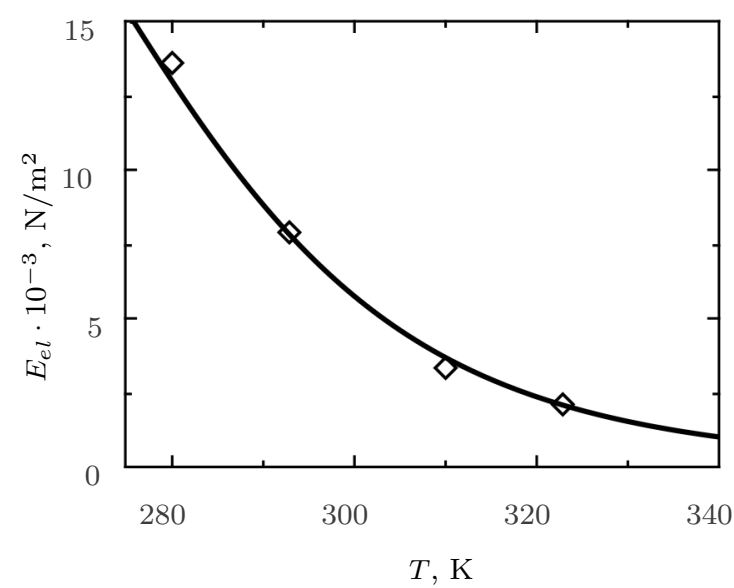

Fig. 7

The elasticity modulus of a free casein gel vs temperature: the points are experimental values, the solid line is an approximation by means of equation (20).

In this case, transition from one temperature to another occurs slowly so that during this time a quasiequilibrium value of the elasticity modulus could be reached. That is, starting from equation (17), we can assume that $d E_{e l} / d t=0$. Writing this condition in both cases, we obtain an equation relating $U_{s}, K_{r}$ and $E_{0}$ :

$E_{e l}=\frac{K_{r} E_{0}^{5 / 3} T}{K_{r} E_{0}^{2 / 3} T+\nu_{f}}$.

Using the value of $\tau_{0}=10^{-12} \mathrm{~s}$ and equation (20), we determine the values of $U_{s}, K_{r}$ and $E_{0}$, which allow us to approximate the experimental values of $E_{e l}$ shown in the Table 1 and in Figure 7 . The obtained values are $U_{s}=0.45 \mathrm{eV}, K_{r}=0.023 \mathrm{~m}^{4 / 3} \mathrm{~K}^{-1} \mathrm{~s}^{-1} \mathrm{~N}^{-2 / 3}$ and $E_{0}=3.0 \cdot 10^{4} \mathrm{~N} / \mathrm{m}^{2}$, which is the maximum possible value of the elasticity modulus for a free gel. In what follows we assume that the value $K_{r}$ is the same for both the free gel and the polymer adlayer of nanoparticles. However, unlike a free gel, the $E_{0}$ value for a nanoparticle adlayer should be much larger. It can be found using an equation similar to equation (20) if instead of $E_{e l}$ we take the value of the elasticity modulus determined in the previous Section 2 from the condition of balance of forces at room temperature $T_{r}=300 \mathrm{~K}$. Taking into account that $E_{e l}\left(T_{r}\right) \geq 10^{5} \mathrm{~N} / \mathrm{m}^{2}$ we obtain for $U_{s}=0.45 \mathrm{eV}$ and $\tau_{0}=10^{-12} \mathrm{~s}$ the following estimate for $E_{0}$ :

$E_{0}=\left(E_{e l}\left(T_{r}\right)^{1 / 3}+1340 / E_{e l}\left(T_{r}\right)^{1 / 3}\right)^{3}$.

Thus the terms of equation (17) are completely defined

$$
\frac{d E_{e l}}{d t}=-\left[\nu_{f}(T)+K_{r} E_{0}^{2 / 3} T\right] \cdot E_{e l}+K_{r} E_{0}^{5 / 3} T .
$$

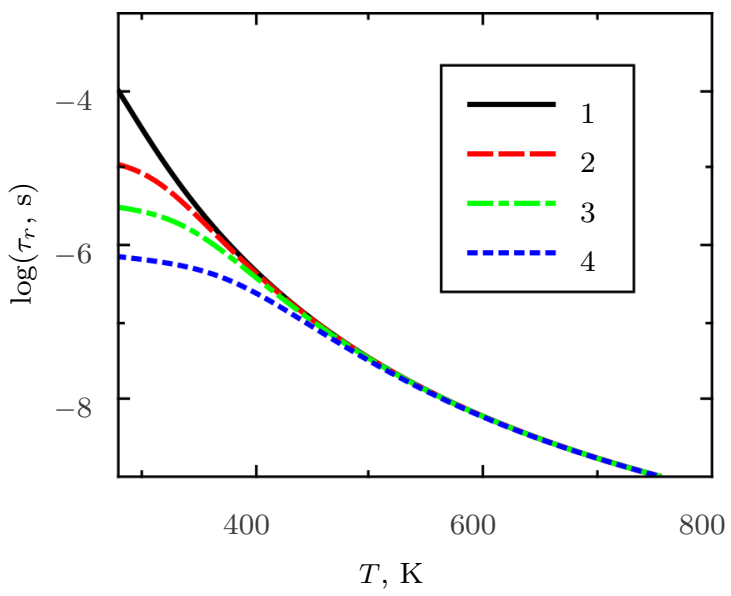

Fig. 8

Dependence of the relaxation time on temperature for different values of $E_{e l}\left(T_{r}\right): 1-5.8 \cdot 10^{3} \mathrm{~N} / \mathrm{m}^{2}, 2-10^{6} \mathrm{~N} / \mathrm{m}^{2}$, $3-10^{7} \mathrm{~N} / \mathrm{m}^{2}, 4-10^{8} \mathrm{~N} / \mathrm{m}^{2}$.

Here the term in brackets determines the characteristic EM relaxation time

$\tau_{r}=\left[\nu_{f}(T)+K_{r} E_{0}^{2 / 3} T\right]^{-1}$,

which depends on temperature and $E_{0}$. Fig. 8 below shows dependences $\tau_{r}(T)$ for different values of $E_{e l}\left(T_{r}\right)$.

We note that the obtained values agree with the characteristic relaxation times $\left(\sim 10^{-1}-10^{-6} \mathrm{~s}\right)$ of polymers in the region of relatively small heating $(T \leq$ $400 \mathrm{~K}$ ) [49] and the temperature dependence has a similar character: the relaxation time increases with decrease in the temperature.

Fig. 8 shows two characteristic parts of the function $\log \left(\tau_{r}\right)$. At low temperatures (from 300 to $350 \mathrm{~K}$ ), the function exhibits a gentle slope corresponding to a power-law dependence $\tau_{r}$ on $T$, which is determined by the second term in brackets in equation (23). Upon temperature growth, the exponential term $\left(\nu_{f}\right)$ in this equation plays a decisive role. It is also clear that as the value of $E_{e l}\left(T_{r}\right)$ increases, the relaxation time of the EM at low temperatures decreases, and the range of the power-law dependence of $\tau_{r}$ grows.

Figure 9 shows an example of calculating variation of the relative value of the elasticity modulus for a given stepwise change in temperature. At the initial temperature jump (the melting stage of the polymer), a rapid $(\sim 0.2 \mu \mathrm{s})$ relaxation of the elastic modulus to the equilibrium value $\left(\sim 0.02 \cdot E_{e l}\left(T_{r}\right)\right)$ occurs at the temperature $500 \mathrm{~K}$. As the temperature drops the elasticity modulus is restored. The lower the temperature, the longer the recovery process. The process of adlayer melting and its reconstruction can play an important role when considering the impact of laser radiation on 


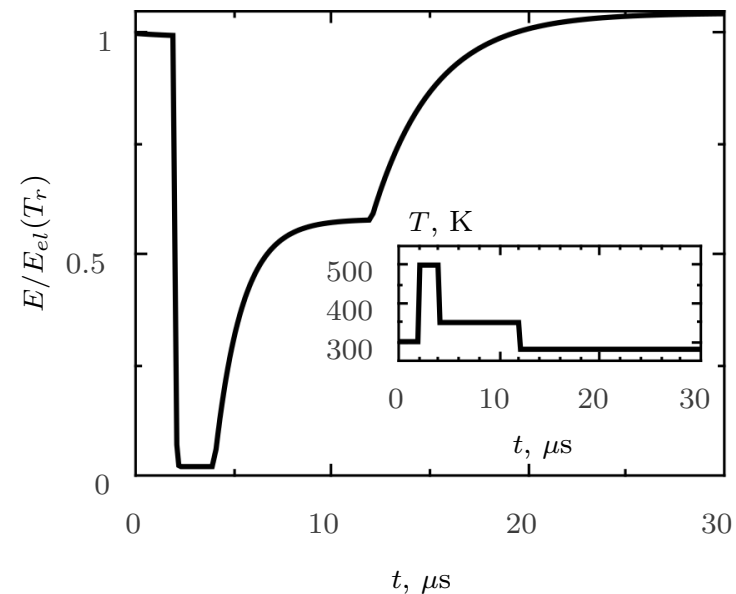

Fig. 9

Relaxation of the elasticity modulus of a polymer layer of a nanoparticle for a given temperature change (graph in the insert) and an initial value $E_{e l}\left(T_{r}\right)=10^{7} \mathrm{~N} / \mathrm{m}^{2}$ at $T_{r}=300 \mathrm{~K}$.

colloid plasmonic nanoparticle aggregates and manifestation of photochromic effects.

\section{Conclusion}

In this paper we have addressed one of the oldest problems of colloid chemistry related to the stability of disperse systems and kinetics of their coagulation which is relevant hitherto. We have proposed a model of pairwise elastic interaction of contacting nanoparticles with a non-deformable core and an elastically deformable polymeric adlayer based on the Hertz deformation problem. Adaptation of the macroscopic Hertz theory to nanoparticles requires strong modification of the elasticity modulus. Its magnitude is determined by balance of the van der Waals and elastic deformation forces. The model was extended to include the temperature dependence of the elasticity modulus. In such nanoparticles the elasticity modulus of the adlayer depends on its thickness and the particle size as well as on temperature and heating rate.

We employed a kinetic approach to take into account temperature dependence of the elastic properties of adlayer. It is based on the assumption of proportionality of the elasticity modulus to the concentration of the nodes of the polymer network and the finite relaxation time of the nodes when the temperature of the adlayer changes. The latter means that the value of the elasticity modulus depends not only on the temperature, but also on the rate of its change. This is especially important when particles are heated by short laser pulses.

The temperature dependence of the elasticity modulus is described by equation (20). Its magnitude can be found from balance of the van der Waals attraction forces and the elastic repulsion of a pair of contacting particles. The energy of elastic repulsion is found by solving the Hertz problem on elastic deformation of two spherical bodies in contact (equation (2) for monodisperse colloids and equation (1) for polydisperse colloids). We have shown that the role of the rigid core is important when its size is comparable with or larger than the thickness of the adsorption layer. We note that the use of the presented model in our works [22, $28,29]$ yielded fairly adequate results on laser photomodification of disordered colloid Ag nanoparticle aggregates, which quite satisfactory reproduce the experimental data, and enabled us to establish the regularities of crystallization of nanocolloids.

An important advantage of the presented models is their ability to provide an adequate description of elastic repulsion of nanoparticles with a polymer adlayer under rapidly changing over a wide range of temperatures using only macroscopic parameters in the absence of information on the characteristics of polymer molecules.

Acknowledgements The reported research was funded by Russian Foundation for Basic Research and the government of the Krasnoyarsk territory, grant 18-42-243023, the RF Ministry of Education and Science, the State contract with Siberian Federal University for scientific research in 20172019 and SB RAS Program No II.2P (0358-2015-0010).

Conflict of Interest The authors confirm that there are no known conflicts of interest associated with this publication.

\section{References}

1. T. Tadros, Suspension Concentrates: Preparation, Stability and Industrial Applications. De Gruyter Textbook (De Gruyter, 2017). URL https://books.google.ru/books? id=9R29DgAAQBA J

2. S.J. Rzoska, S. Starzonek, A.D. Rzoska, in Advances in Colloid Science, ed. by M.M. Rahman, A.M. Asiri (InTech, Rijeka, 2016), chap. 12. DOI 10.5772/64910. URL http://dx.doi.org/10.5772/64910

3. P. Adiseshaiah, J. Clogston, C. Mcleland, J. Rodriguez, A. M Potter, B. Neun, S. Skoczen, S.S. Shanmugavelandy, M. Kester, S. T Stern, S. E McNeil, Cancer letters 337 (2013)

4. B.M. Barth, S.S. Shanmugavelandy, J.M. Kaiser, C. McGovern, J.K. Haakenson, J.A. Hengst, E.L. Gilius, S.A. Knupp, T.E. Fox, J.P. Smith, T.M. Ritty, J.H. Adair, M. Kester, ACS Nano 7(3), 2132 (2013). DOI 10.1021/ nn304862b

5. E. Casals, M.F. Gusta, M. Cobaleda-Siles, A. GarciaSanz, V.F. Puntes, Cancer Nanotechnology 8(1), 7 (2017). DOI 10.1186/s12645-017-0030-4. URL https: //doi.org/10.1186/s12645-017-0030-4 
6. W.J. Parak, D. Gerion, T. Pellegrino, D. Zanchet, C. Micheel, S.C. Williams, R. Boudreau, M.A.L. Gros, C.A. Larabell, A.P. Alivisatos, Nanotechnology 14(7), R15 (2003). URL http://stacks.iop.org/0957-4484/14/ $i=7 / a=201$

7. J. Wang, W.J. Blau, Journal of Optics A: Pure and Applied Optics 11(2), 024001 (2009). DOI 10.1088/ 1464-4258/11/2/024001. URL https://doi.org/10.1088/ $1464-4258 / 11 / 2 / 024001$

8. Y.P. Sun, J.E. Riggs, K.B. Henbest, R.B. Martin, Journal of Nonlinear Optical Physics \& Materials 09(04), 481 (2000). DOI 10.1142/s0218863500000315. URL https://doi.org/10.1142/s0218863500000315

9. L. Irimpan, V.P.N. Nampoori, P. Radhakrishnan, B. Krishnan, A. Deepthy, Journal of Applied Physics 103(3), 033105 (2008). DOI 10.1063/1.2838178. URL https: //doi.org/10.1063/1.2838178

10. N. Board, The Complete Technology Book on Printing Inks (Asia Pacific Business Press, 2003). URL https://books. google.ru/books?id=apZgCwAAQBAJ

11. D. Guo, G. Xie, J. Luo, Journal of Physics D: Applied Physics 47(1), 013001 (2014). URL http://stacks.iop. org $/ 0022-3727 / 47 / i=1 / a=013001$

12. U. Kreibig, M. Vollmer, Optical Properties of Metal Clusters (Springer-Verlag, Berlin, 1995)

13. B.V. Deryagin, Theory of Stability of Colloids and Thin Films (Consultants Bureau, New York, 1989)

14. J.N. Israelachvili, Intermolecular And Surface Forces: With Applications To Colloidal And Biological Systems (Academic Press, London, 1992)

15. H. Pletsch, Design of elastic nanocomposite materials from polymers and noble metal nanoparticles. Ph.D. thesis, Bayreuth (2015). URL https://epub.uni-bayreuth. de/3184/

16. S.E. Harton, S.K. Kumar, H. Yang, T. Koga, K. Hicks, H. Lee, J. Mijovic, M. Liu, R.S. Vallery, D.W. Gidley, Macromolecules 43(7), 3415 (2010). DOI 10.1021/ ma902484d

17. D.J. Goodwin, S. Sepassi, S.M. King, S.J. Holland, L.G. Martini, M.J. Lawrence, Molecular Pharmaceutics 10(11), 4146 (2013). DOI 10.1021/mp400138e

18. P.J. Griffin, V. Bocharova, L.R. Middleton, R.J. Composto, N. Clarke, K.S. Schweizer, K.I. Winey, ACS Macro Letters 5(10), 1141 (2016). DOI 10.1021/acsmacrolett. $6 \mathrm{~b} 00649$

19. S.V. Karpov, V.V. Slabko, Optical and Photophysical Properties of Fractal-Structured Metal Sols (Russian Academy of Sciences, Siberian Branch, Novosibirsk, 2003)

20. S.V. Karpov, M.K. Kodirov, A.I. Ryasiyanskiy, V.V. Slabko, Quantum Electronics 31(10), 904 (2001)

21. A.E. Ershov, I.L. Isaev, P.N. Semina, V.A. Markel, S.V. Karpov, Physical Review B 85, 045421 (2012)

22. S.V. Karpov, I.L. Isaev, A.P. Gavrilyuk, V.S. Gerasimov, G.A. S., Colloid journal 71(3), 314 (2009)

23. D. Werner, S. Hashimoto, T. Uwada, Langmuir 26(12), 9956 (2010). DOI 10.1021/la100015t. URL https://doi. org/10.1021/la100015t

24. V.P. Safonov, V.M. Shalaev, V.A. Markel, Y.E. Danilova, N.N. Lepeshkin, W. Kim, S.G. Rautian, R.L. Armstrong, Physical Review Letters 80(5), 1102 (1998)

25. V.P. Drachev, S.V. Perminov, S.G. Rautian, V.P. Safonov, in Topics in Applied Physics (Springer Berlin Heidelberg, 2002), pp. 115148. DOI $10.1007 / 3-540-44948-5 \_6$. URL https://doi.org/10.1007/3-540-44948-5_6
26. N.N. Lepeshkin, Optical properties of metal-dielectric composites. Ph.D. thesis, New Mexico State University (2001)

27. V.P. Drachev, D.W. Bragg, V.A. Podolskiy, V.P. Safonov, W.T. Kim, Z.C. Ying, R.L. Armstrong, V.M. Shalaev, JOSA B 18(12), 1896 (2001)

28. A.P. Gavrilyuk, S.V. Karpov, Appl. Phys. B 97, 163 (2009)

29. A.E. Ershov, A.P. Gavrilyuk, , P.N. Semina, S.V. Karpov, P.N. Semina, Appl. Phys. B. 115, 547 (2014)

30. A. Jones, B. Vincent, Colloids and Surfaces 42, 113 (1989)

31. R.G. Uttarwar, J. Potoff, Y. Huang, Industrial \& Engineering Chemistry Research 52(1), 73 (2013). DOI 10.1021/ie301228f

32. L. Di Michele, A. Zaccone, E. Eiser, Proceedings of the National Academy of Sciences 109(26), 10187 (2012). DOI 10.1073/pnas.1202171109. URL http://www.pnas. org/content/109/26/10187. abstract

33. B. Vincent, J. Edwards, S. Emmett, A. Jones, Colloids and Surfaces 18, 261 (1986)

34. J.A. Lewis, Journal of the American Ceramic Society 83, $2341(2000)$

35. K. Lu, Ceramics International 34(6), 1353 (2008). DOI https://doi.org/10.1016/j.ceramint.2007.02.016. URL http://www.sciencedirect.com/science/article/pii/ S0272884207001010

36. L. Landau, E. Lifshitz, A. Kosevich, L. Pitaevski, Theory of Elasticity. Course of theoretical physics (ButterworthHeinemann, 1986). URL https://books.google.ru/ books?id=tpY-VkwCkAIC

37. H. Hamaker, Physica 4(10), 1058 (1937). DOI 10.1016/ s0031-8914(37)80203-7. URL https://doi.org/10.1016/ s0031-8914(37) 80203-7

38. H. Sonntag, K. Strenge, Koagulation und Stabilitatdisperser Systeme (VEB Deutscher Verlag der Wissenschaften, Berlin, 1970)

39. S.V. Karpov, V.V. Slabko, G.A. Chiganova, Colloid J. 64(4), 425 (2002)

40. M.N. Pankratova, V.N. Izmailova, Colloid Journal of the USSR 38, 446 (1976)

41. G.M. Bartenev, S. Frenkel, Physics of Polymers (Khimia, Leningrad, 1990)

42. E. Dill, The Finite Element Method for Mechanics of Solids with ANSYS Applications. Advances in Engineering Series (CRC Press, 2011)

43. V.N. Izmailova, G.P. Yampolskaya, B.D. Summ, Surface Phenomena in Protein Systems (Khimia, Moscow, 1988)

44. G.M. Bartenev, U.V. Zelenev, Physics and mechanics of Polymers (Visshaya Shkola, Moscow, 1983)

45. V.R. Regel, A.I. Slutsker, E.E. Tomashevskii, Phys. Usp. 15(1), 45 (1972). DOI 10.1070/ PU1972v015n01ABEH004945. URL https: //ufn.ru/en/articles/1972/1/c/

46. H.H. Kausch von Schmeling, S.R. Moghe, C.C. Hsiao, Journal of Applied Physics 38(1), 201 (1967). DOI 10. 1063/1.1708955

47. G. Rehage, Pure and Applied Chemistry 39(1-2), 161 (1974). DOI 10.1351/pac197439010161. URL https:// doi.org/10.1351/pac197439010161

48. J. Frenkel, Kinetic Theory of Liquids. Dover Publications (Dover, 1955). URL https://books.google.ru/books?id= ORdSQwAACAAJ

49. A.A. Tager, Physical Chemistry of Polymers (MIR Publishers, Moscow, 1978) 